\title{
Collaborative care for anxiety disorders in primary care: a systematic review and meta-analysis
}

\author{
Anna DT Muntingh ${ }^{1 *}$, Christina M van der Feltz-Cornelis ${ }^{2,3}$, Harm WJ van Marwijk ${ }^{4,5}$, Philip Spinhoven ${ }^{6,7}$ \\ and Anton JLM van Balkom ${ }^{1}$
}

\begin{abstract}
Background: Studies evaluating collaborative care for anxiety disorders are recently emerging. A systematic review and meta-analysis to estimate the effect of collaborative care for adult patients with anxiety disorders in primary care is therefore warranted.

Methods: A literature search was performed. Data sources: PubMed, Psycinfo, Embase, Cinahl, and the Cochrane library. Study eligibility criteria: Randomized controlled trials examining the effects of collaborative care for adult primary care patients with an anxiety disorder, compared to care as usual or another intervention. Synthesis methods: Standardized mean differences (SMD) on an anxiety scale closest to twelve months follow-up were calculated and pooled in a random effects meta-analysis.

Results: Of the 3073 studies found, seven studies were included with a total of 2105 participants. Included studies were of moderate to high quality. Collaborative care was superior to care as usual, with a small effect size $(S M D=0.35$ $95 \% \mathrm{Cl} 0.14-0.56)$ for all anxiety disorders combined and a moderate effect size (SMD $=0.59,95 \% \mathrm{Cl} 0.41-0.78)$ in a subgroup analysis (five studies) on patients with panic disorder.

Conclusions: Collaborative care seems to be a promising strategy for improving primary care for anxiety disorders, in particular panic disorder. However, the number of studies is still small and further research is needed to evaluate the effectiveness in other anxiety disorders.
\end{abstract}

Keywords: Collaborative care, Primary care, Anxiety disorders, Systematic review, Meta-analysis, Randomized controlled trials

\section{Background}

Anxiety disorders constitute the most prevalent category of psychiatric disorders [1]. Anxiety disorders have a negative impact on quality of life and are associated with significant healthcare- and productivity costs [2]. Adults with an anxiety disorder mainly receive care in primary care [3-5]. In many countries however, the quality of care for adults with anxiety disorders leaves room for improvement [4, 6]. Although clinical guidelines recommend cognitive behavioral therapy $(\mathrm{CBT})$ or antidepressant medication as the treatment of choice in primary care, these evidence-

\footnotetext{
*Correspondence: a.muntingh@ggzingeest.nl

'Department of Psychiatry, VU University Medical Center / GGZ inGeest, A.J. Ernststraat 1187, Amsterdam $1081 \mathrm{HL}$, The Netherlands

Full list of author information is available at the end of the article
}

based treatments are not often adequately applied in primary care [7].

Several barriers still exist in providing evidence-based care for anxiety disorders in primary care, which may be related to patient characteristics, provider characteristics or the organizational context of primary care [8-11]. Therefore, multifaceted interventions that focus on patient, provider and organization of care have been proposed as the most promising strategy to improve primary mental healthcare [12, 13]. Collaborative care models are such multifaceted interventions, bringing mental health expertise into primary care by introducing new members into the primary care team. Typically, this new member is a "care manager" - a mental health professional who coordinates care, provides evidence-based interventions, and actively 
monitors the patients' symptoms [14]. The care manager usually is a non-physician professional, such as a psychologist, a social worker or a psychiatric nurse. The care manager works in close collaboration with the primary care physician and preferably providers have access to the tailored advice of a psychiatrist. Collaborative care models often vary in types of interventions used, the intensity of treatment, collaboration, and follow-up [15]. However, some essential elements have been described by experts and in systematic reviews, which consider cooperation between the primary care physician and at least one other professional, provision of evidence-based treatment, and active monitoring of symptoms [14, 16-18]. Interventions or organizational models similar to collaborative care are sometimes referred to as integrated care, enhanced care, or care management.

\section{Rationale}

Previous reviews on collaborative care included only a limited number of studies on anxiety disorders (4 in the review of Archer and colleagues [19] and 3 in the review of Woltmann and colleagues [20]). Furthermore, all of these studies were conducted in the United States, which may limit generalizability to primary care in other countries. We are furthermore aware of recent studies $[21,22]$ in other countries, so we systematically reviewed the literature on collaborative (primary) care for anxiety disorders, again.

Evidence of the effectiveness of collaborative care in the treatment of depression is well established and was reviewed thoroughly in several meta-analyses [19, $20,23,24]$. The field has progressed to identifying elements of collaborative care that contribute most to its effectiveness. Recently, Coventry and colleagues [25] concluded that the provision of a psychological intervention increases the effectiveness of collaborative care based on a meta-regression. We were curious to what extent and how this could also apply to anxiety disorders.

\section{Objectives}

We performed a systematic review and meta-analysis to summarize results from randomized controlled trials about the effectiveness of collaborative care for anxiety disorders in adult primary care patients compared to care as usual. In addition, we evaluated the effects for specific anxiety disorders and the influence of several characteristics of study procedures and collaborative care interventions on the effectiveness of the collaborative care model. We used the PRISMA checklist for reporting systematic reviews [26].

\section{Methods \\ Eligibility criteria \\ Design and population}

We included published, randomized controlled trials (RCTs) that evaluated collaborative care compared to care as usual or another active intervention in adult primary care patients with an anxiety disorder and that reported outcomes on a standardized scale for anxiety severity. Both individually randomized and cluster randomized trials were included.

Studies had to include adult ( $>18$ years) subjects recruited in a primary care setting with an anxiety disorder as established with a valid diagnostic interview, according to research diagnostic criteria, or with a cut-off score on a validated scale. Comorbid medical or psychiatric conditions were allowed, as long as the intervention focused on the anxiety disorder.

\section{Intervention and comparison intervention}

Collaborative care interventions were defined by the application of criterion 1 in combination with criterion 2 and/ or $3[14,16-18]$ :

1. The primary care physician is supported by at least one other professional with a different field of expertise (e.g. care manager, consultant psychiatrist), and they work together in providing care for the patient.

2. Evidence-based treatment is provided.

3. Process and outcome of treatment is monitored.

Studies evaluating the provision of services by an on-site mental health professional were excluded, unless reference was made to enhanced collaboration between the primary care physician and the mental health professional. Collaboration between professionals had to be ensured by team meetings, consultation or supervision, or a digital communication system. The collaborative care intervention could be compared to care as usual, a waitlist condition, or another active intervention.

\section{Outcomes}

Studies that reported outcomes at follow-up closest to 12 months on a validated continuous anxiety scale or dichotomous interview (indicating response or remission) were included. Standardized scales or interviews could measure general anxiety (across anxiety disorders) or measure a specific type of anxiety (e.g. panic disorder severity).

\section{Search strategy and selection criteria}

We searched PubMed (Medline), Psycinfo, Embase, The Cochrane Central Register of Controlled Trials, and Cinahl from inception to March $10^{\text {th }} 2014$ without language restriction. The highly sensitive search was performed by 
an experienced librarian and author A.M., combining terms related to anxiety, primary care, and randomized controlled trials. For Medline, we used free text words and $\mathrm{MeSH}$ terms such as "Anxiety", "Anxiety Disorders", "Primary Health Care" and "Family Practice". We combined these with a pre-tested search string for randomized controlled trials. See Fig. 1 for the full search strategy as performed in PubMed, which was adapted for use in the other databases. The reference lists of selected randomized controlled trials
(RCTs) and reviews were checked for potentially relevant titles. The search was limited to published studies.

\section{Study selection}

Titles and abstracts of retrieved studies were screened independently by two reviewers (AM/CFC) using a list of inclusion criteria. If a study appeared eligible (or if eligibility was doubtful), the full text of an article was retrieved. All fulltext articles were assessed for eligibility by two independent

\begin{tabular}{|c|c|c|c|}
\hline Search & Most Recent Queries & Time & Result \\
\hline$\# 12$ & Search \#9 OR \#10 OR \#11 & 08:53:48 & $\underline{955}$ \\
\hline \#11 & Search \#7 Limits: Systematic Reviews & | $08: 49: 18$ & $\underline{196}$ \\
\hline$\# 10$ & $\begin{array}{l}\text { Search \#7 Limits: Clinical Trial, Meta-Analysis, Practice Guideline, Randomized } \\
\text { Controlled Trial }\end{array}$ & 08:49:04 & $\underline{727}$ \\
\hline$\# 9$ & Search (\#7 AND \#8) NOT medline[sb] & || $08: 48: 29$ & 111 \\
\hline \#8 & \begin{tabular}{|l} 
Search randomized controlled trial [pt] OR controlled clinical trial [pt] OR clinical trial \\
[pt] OR comparative study [pt] OR evaluation studies [pt] OR "randomized controlled \\
trials as topic"[MeSH Terms] OR "random allocation"[MeSH Terms] OR "double-blind \\
method"[MeSH Terms] OR "single-blind method"[MeSH Terms] OR "clinical trials as \\
topic"[MeSH Terms] OR "placebos"[MeSH Terms] OR "research design"[MeSH \\
Terms:noexp] OR "follow-up studies"[MeSH Terms] OR "prospective studies"[MeSH \\
Terms] OR "cross-over studies"[MeSH Terms] OR "drug therapy"[Subheading] OR \\
"clinical trial" [tw] OR "latin square" [tw] OR placebo* [tw] OR random* [tw] OR \\
control[tw] OR controll*[tw] OR prospectiv*[tw] OR volunteer* [tw] OR trial[tiab] OR \\
groups[tiab] OR ((singl* [tw] OR doubl* [tw] OR trebl* [tw] OR tripI* [tw]) AND (mask* \\
[tw] OR blind* [tw]))
\end{tabular} & 08:48:11 & 6213954 \\
\hline$\# 7$ & Search \#3 AND \#6 & || $08: 47: 36$ & 4922 \\
\hline \#6 & Search \#4 OR \#5 & || $08: 47: 24$ & 185546 \\
\hline$\# \underline{7}$ & Search "Primary Health Care"[Mesh] OR "primary care"[tiab] & |08:47:11 & 91683 \\
\hline$\# 4$ & \begin{tabular}{|l} 
Search ("Family Practice"[Mesh] OR "Physicians, Family"[Mesh]) OR "family \\
practice"[tiab] OR "general practice"[tiab] OR"family practices"[tiab] OR "general \\
practices"[tiab] OR "family practitioner "[tiab] OR "general practitioner"[tiab] OR \\
"family practitioners "[tiab] OR "general practitioners"[tiab] OR family medicine[tiab] \\
OR "Physician Assistants"[Mesh] OR "Physician Assistant"[tiab] OR "Physician \\
Assistants"[tiab] OR "Nurse Practitioners"[Mesh] OR "Nurse Practitioner"[tiab] OR \\
"Nurse Practitioners"[tiab]
\end{tabular} & $\mid 08: 46: 55$ & 115493 \\
\hline$\# \underline{3}$ & Search \#1 OR \#2 & $\mid 08: 46: 08$ & 149816 \\
\hline$\# 2$ & \begin{tabular}{|l} 
Search Anxiety[tiab] OR Anxieties[tiab] OR anxious[tiab] OR Nervousness[tiab] OR \\
Agoraphobia*[tiab] OR Obsessive-Compulsive[tiab] OR Panic*[tiab] OR Phobia*[tiab] \\
OR Phobic*[tiab] OR Claustrophobi*[tiab] OR (Stress[tiab] AND trauma*[tiab]) OR \\
(Stress[tiab] AND posttrauma*[tiab])
\end{tabular} & |08:45:59 & 118672 \\
\hline$\# 1$ & Search "Anxiety"[Mesh:noexp] OR "Anxiety Disorders"[Mesh] & $08: 45: 16$ & $\underline{92083}$ \\
\hline
\end{tabular}

Fig. 1 PubMed Search History for randomized controlled trials examining the effects of collaborative care for adult primary care patients with an anxiety disorder, compared to care as usual or another intervention 
reviewers ( $\mathrm{AM}$ and $\mathrm{AvB} / \mathrm{HvM} / \mathrm{CFC}$ ). Disagreement was resolved by consensus or a third reviewer. The outcome data were extracted by two reviewers independently (AM and $\mathrm{CFC}$ ). Other relevant characteristics of studies were extracted by one author (AM) using a form based on Cochrane criteria [27] (see Table 1).

\section{Data collection process}

We extracted sample size, means, and standard deviations for scores on anxiety scales at baseline and follow-up for the intervention group and the control group. Outcomes for anxiety disorders in general as well as outcomes for specific anxiety disorders were extracted. For studies using more than one validated anxiety scale as an outcome measure, we chose the reported primary outcome measure. If the mean and standard deviation were not reported, we searched for other data necessary to calculate an effect size, such as a difference score with a standard deviation or confidence limits, and $p$-value [27]. Furthermore, data relevant for the interpretation of the findings such as the setting, diagnoses, the interventions used and the professionals involved were collected (see Table 1). Where published protocols of the studies included were available, they were used to supplement data about intervention details.

\section{Risk of bias in individual studies}

The risk of bias of each included study was assessed using a standard form based on Cochrane criteria [28] by two reviewers ( $\mathrm{AM}$ and $\mathrm{AvB} / \mathrm{HvM}$ ) independently. The form systematically enquired about possible sources of bias in randomized controlled trials, such as the adequacy of the randomization procedure, allocation concealment, handling of missing data and selective reporting. Disagreement between reviewers about assessment ratings were resolved by consensus or a third reviewer (CFC).

\section{Synthesis of results}

We statistically summarized the effectiveness of collaborative care interventions versus the comparison interventions using meta-analysis. The analyses were conducted using the software package Comprehensive Meta Analysis version 2.0 [29]. We calculated a standardized mean difference (SMD) from reported differences in means on a continuous anxiety scale between interventions at 12 months followup. We summarized the SMDs using the random effects model [30]. To assess the heterogeneity among studies, we calculated the $I^{2}$ statistic, which reflects the proportion of total variation across studies that is attributable to heterogeneity rather than chance. An $I^{2}$ of $0 \%$ means that there is no observed heterogeneity, while an $I^{2}$ of 25,50 and $75 \%$ may be interpreted as low, medium, and high heterogeneity respectively [31].

\section{Risk of bias across studies}

Funnel plots were created and Duval and Tweedie's trim and fill method was used to examine the possibility of publication bias [32]. This method gives an estimate of the effect size after correcting for possible publication bias.

\section{Additional analyses}

A predefined subgroup analysis was performed to assess the effectiveness of collaborative care for patients with a specific anxiety disorder. A disorder-specific outcome measure (if available) was used to calculate the effect size in a random effects meta-analysis. In an additional subgroup analysis, we examined several variables related to study procedures and intervention details based on the meta-regression of Coventry and colleagues [25]. We omitted variables described by Coventry and colleagues that were not present in any of the included studies and added the element of stepped care as this was a prominent aspect of studies 6 and 7 [21, 22]. For this subgroup analysis, we used a mixed-effects model which pools studies in a subgroup using a random effects model. In addition, we used a fixed-effects model to interpret variance between studies $\left(\mathrm{Q}\right.$ and $\left.\mathrm{I}^{2}\right)$ and to test for significant differences between subgroups.

\section{Results}

\section{Study selection}

The literature search resulted in a total of 4929 retrieved citations. After removal of duplicates, 3073 abstracts were available (see Fig. 2). Main reasons for exclusion of studies based on titles or abstracts were no RCT, no inclusion of anxiety disorders and no collaborative care. For 20 studies the full-text paper was retrieved and examined for inclusion. After the exclusion of 13 studies (nine no collaborative care; three no separate outcome reported for patients with anxiety disorders; one report of other study), there were seven studies that met all inclusion criteria.

\section{Study characteristics}

Seven studies involving 2105 participants (1107 in the collaborative care condition, 998 in the control condition) were included: Roy-Byrne et al. 2001 [33] (study 1); RoyByrne et al. 2005 [34] (study 2); Rollman et al. 2005 [35] (study 3); König et al. 2009 [36] (study 4); Roy-Byrne et al. 2010 [37] (study 5); Oosterbaan et al. 2013 [21] (study 6); and Muntingh et al. 2014 [22] (study 7). Table 1 shows an overview of characteristics of the included studies.

\section{Design and participants}

The number of participants in each study ranged from 57 to 1004 . Of the trials, four were individually randomized controlled trials (study 1-3, 5); three used cluster randomization on the level of primary care practices (study 4, 6, 7); four were conducted in the USA (studies 
Table 1 Characteristics of randomized controlled trials comparing collaborative care for anxiety disorders with care as usual

\begin{tabular}{|c|c|c|c|c|c|c|c|c|c|c|c|c|c|}
\hline $\begin{array}{l}\text { Study } \\
\text { No. }\end{array}$ & $\begin{array}{l}\text { Authors/ } \\
\text { year }\end{array}$ & De-sign & Recruit-ment & $\begin{array}{l}\text { Diagn } \\
\text { instr. }\end{array}$ & Int. & Setting & $\begin{array}{l}N \\
(\mathrm{ITT})\end{array}$ & $\begin{array}{l}\text { Collaborative care } \\
\text { intervention }\end{array}$ & $\begin{array}{l}\text { Professionals } \\
\text { involved }\end{array}$ & $\begin{array}{l}\text { Comparison } \\
\text { intervention }\end{array}$ & Outcomes FU & $\begin{array}{l}\text { Outcome CC } \\
\text { vs CAU }\end{array}$ & $\begin{array}{l}\text { Outcome at } \\
12 \text { months } \\
{[95 \% \mathrm{Cl}]}\end{array}$ \\
\hline 1 & $\begin{array}{l}\text { Roy-Byrne } \\
\text { et al. 2001 } \\
\text { (Study 1) }\end{array}$ & $\mathrm{RCT}$ & $\begin{array}{l}\text { Referral } \\
\text { Screening } \\
\text { (waiting } \\
\text { room, } \\
\text { PHQ-2 PD) }\end{array}$ & $\begin{array}{l}\mathrm{PD} \\
\mathrm{CIDI}\end{array}$ & $\begin{array}{l}\text { CC } \\
\text { VS } \\
\text { CAU }\end{array}$ & $\begin{array}{l}3 \text { primary } \\
\text { care } \\
\text { clinics } \\
\text { (US) }\end{array}$ & $\begin{array}{l}\text { CC: } \\
57 \\
\text { CAU: } \\
58\end{array}$ & $\begin{array}{l}\text { Medication } \\
\text { management by } \\
\text { psychiatrist }\end{array}$ & $\begin{array}{l}\mathrm{PCP} \\
\text { psychiatrist }\end{array}$ & $\begin{array}{l}\text { CAU by PCP, i.e. } \\
\text { pharmacotherapy } \\
\text { or referral to mental } \\
\text { health professional }\end{array}$ & $\begin{array}{l}\text { ASI, PDSS } \\
3,6,9,12 \\
\text { months }\end{array}$ & $\begin{array}{l}\text {-Improved } \\
\text { anxiety } \\
\text { outcome } \\
\text { at } 3,6 \text { and } \\
12 \text { months } \\
\text {-Improved } \\
\text { panic outcome } \\
\text { at } 6 \text { months }\end{array}$ & $\begin{array}{l}\text {-Anxiety/panic (ASI): } \\
T=2.14, p=0.035 \\
\text { ES 0.45 [0.03-0.87] } \\
\text {-Panic (PDSS): } \\
\text { statistics continuous } \\
\text { outcome not reported }\end{array}$ \\
\hline 2 & $\begin{array}{l}\text { Roy-Byrne } \\
\text { et al. 2005 } \\
\text { (Study 2) }\end{array}$ & $\mathrm{RCT}$ & $\begin{array}{l}\text { Referral } \\
\text { Screening } \\
\text { (waiting } \\
\text { room, } \\
\text { PHQ-2 PD) }\end{array}$ & $\begin{array}{l}\text { PD } \\
\text { CIDI }\end{array}$ & $\begin{array}{l}\text { CC } \\
\text { VS } \\
\text { CAU }\end{array}$ & $\begin{array}{l}\text { University } \\
\text { affiliated } \\
\text { primary } \\
\text { care } \\
\text { clinics } \\
\text { (US) }\end{array}$ & $\begin{array}{l}\text { CC: } \\
119 \\
\text { CAU: } \\
113\end{array}$ & $\begin{array}{l}\text { CBT and/or } \\
\text { antidepressant } \\
\text { medication }\end{array}$ & $\begin{array}{l}\text { PCP, CM, } \\
\text { psychiatrist }\end{array}$ & $\begin{array}{l}\text { CAU by PCP, i.e. } \\
\text { pharmacotherapy } \\
\text { or referral to mental } \\
\text { health professional }\end{array}$ & $\begin{array}{l}\text { ASI } \\
3,6,9,12 \\
\text { months }\end{array}$ & $\begin{array}{l}\text {-Improved } \\
\text { anxiety/panic } \\
\text { outcome at all } \\
\text { time points }\end{array}$ & $\begin{array}{l}\text { - Anxiety/panic (ASI): } \\
\text { Dif }-6.64 \\
{[-10.73 \text { to }-2.48]} \\
p<0.001 ; \\
\text { ES } 0.48[0.18-0.78]\end{array}$ \\
\hline 3 & $\begin{array}{l}\text { Rollman et } \\
\text { al. } 2005 \\
\text { (Study 3) }\end{array}$ & $\mathrm{RCT}$ & $\begin{array}{l}\text { Screening } \\
\text { (waiting } \\
\text { room, PHQ) }\end{array}$ & $\begin{array}{l}\text { PD/ } \\
\text { GAD } \\
\text { PRIME- } \\
\text { MD }\end{array}$ & $\begin{array}{l}\text { CC } \\
\text { VS } \\
\text { CAU }\end{array}$ & $\begin{array}{l}4 \\
\text { university } \\
\text { affiliated } \\
\text { primary } \\
\text { care } \\
\text { practices } \\
\text { (US) }\end{array}$ & $\begin{array}{l}\text { CC: } \\
116 \\
\text { CC: } \\
75\end{array}$ & $\begin{array}{l}\text { Guided selfhelp } \\
\text { and/or } \\
\text { antidepressant } \\
\text { medication and/ } \\
\text { or referral to } \\
\text { mental health } \\
\text { specialist }\end{array}$ & $\begin{array}{l}\text { PCP, CM, } \\
\text { psychiatrist/ } \\
\text { Psycho- } \\
\text { therapist }\end{array}$ & $\begin{array}{l}\text { CAU by PCP and } \\
\text { patients received a } \\
\text { diagnosis specific } \\
\text { brochure }\end{array}$ & $\begin{array}{l}\text { SIGH-A, } \\
\text { PDSS } \\
2,4,8,12 \\
\text { months }\end{array}$ & $\begin{array}{l}\text {-Improved } \\
\text { anxiety } \\
\text { outcome at } \\
12 \text { months } \\
\text { - Improved } \\
\text { panic } \\
\text { outcomes at } \\
12 \text { months } \\
\text {-No sign. } \\
\text { improvement } \\
\text { in GAD } \\
\text { outcomes }\end{array}$ & $\begin{array}{l}\text {-Anxiety (SIGH-A): } \\
\text { Dif }-3.6[-6.4 \text { to }-0.8] \\
p=0.01 ; \\
\text { ES 0.43 [O.10-0.77] } \\
\text {-Panic (PDSS): } \\
\text { Diff }-3.3[-5.5 \text { to }-1.1] \\
p=0.004 ; \\
\text { ES 0.58 [0.19-0.97] } \\
- \text { GAD (SIGH-A): } \\
\text { Diff }-1.1[-5.0 \text { to } 2.7] \\
p=0.57 ; \\
\text { ES } 0.13[-0.32 \text { to } 0.58]\end{array}$ \\
\hline 4 & $\begin{array}{l}\text { Konig et al. } \\
2009 \\
\text { (Study 4) }\end{array}$ & $\begin{array}{l}\text { Clus-ter } \\
\mathrm{RCT}\end{array}$ & $\begin{array}{l}\text { Screening } \\
\text { (PHQ) }\end{array}$ & $\begin{array}{l}\mathrm{PD} / \\
\mathrm{GAD} / \\
\text { any } \\
\mathrm{AD} \\
\mathrm{PHQ}\end{array}$ & $\begin{array}{l}\text { CC } \\
\text { VS } \\
\text { CAU }\end{array}$ & $\begin{array}{l}46 \\
\text { primary } \\
\text { care } \\
\text { practices } \\
\text { (GER) }\end{array}$ & $\begin{array}{l}\text { CC: } \\
201 \\
\text { CAU: } \\
188\end{array}$ & $\begin{array}{l}\text { Counselling (CBT) } \\
\text { by the PCP }\end{array}$ & $\begin{array}{l}\text { PCP, } \\
\text { psychiatrist/ } \\
\text { Psycho- } \\
\text { therapist }\end{array}$ & $\begin{array}{l}\text { CAU by PCP, including } \\
\text { referral to mental } \\
\text { health professional }\end{array}$ & $\begin{array}{l}\text { BAl } \\
6,9 \text { months }\end{array}$ & $\begin{array}{l}\text { - No difference } \\
\text { in anxiety } \\
\text { outcomes }\end{array}$ & $\begin{array}{l}\text {-Anxiety (BAI): } \\
\text { CC: M 18.18 SD } 12.17 \\
\text { CAU: M 16.72 SD } 10.34 \\
p=0.35 ; \\
\text { ES }-0.13 \text { [-0.36-0.10] }\end{array}$ \\
\hline 5 & $\begin{array}{l}\text { Roy-Byrne } \\
\text { et al. 2010 } \\
\text { (Study 5) }\end{array}$ & $\mathrm{RCT}$ & Referral & $\begin{array}{l}\text { PD/ } \\
\text { GAD/ } \\
\text { SOP/ } \\
\text { PTSD } \\
\text { MINI }\end{array}$ & $\begin{array}{l}\text { CC } \\
\text { VS } \\
\text { CAU }\end{array}$ & $\begin{array}{l}17 \\
\text { primary } \\
\text { care } \\
\text { clinics } \\
\text { (US) }\end{array}$ & $\begin{array}{l}\text { CC: } \\
503 \\
\text { CAU: } \\
501\end{array}$ & $\begin{array}{l}\text { CBT and/or } \\
\text { antidepressant } \\
\text { medication }\end{array}$ & $\begin{array}{l}\text { PCP, CM, } \\
\text { psychiatrist }\end{array}$ & $\begin{array}{l}\text { CAU by PCP, i.e. } \\
\text { medication, } \\
\text { counseling or referral } \\
\text { to mental health } \\
\text { professional }\end{array}$ & $\begin{array}{l}\text { BSI, PDSS, } \\
\text { GADSS, SPIN, } \\
\text { PCL } \\
6,12,18 \text { months }\end{array}$ & $\begin{array}{l}\text {-Improved } \\
\text { anxiety } \\
\text { outcome at all } \\
\text { time points } \\
\text {-Improved } \\
\text { panic outcome } \\
\text { at } 6 \text { and } \\
12 \text { months } \\
\text {-Improved } \\
\text { GAD outcome } \\
\text { at all time } \\
\text { points } \\
\text {-Improved SOP } \\
\text { outcome at } 6\end{array}$ & $\begin{array}{l}\text {-Anxiety (BSI): } \\
\text { Diff }-2.63[-3.73 \\
\text { to }-1.54] p<0.001 ; \\
\text { ES 0.33 [0.19-0.47] } \\
\text {-Panic (PDSS): } \\
\text { Diff - } 2.71[-4.29 \\
\text { to }-1.14] p=.003 ; \\
\text { ES 0.48 [0.20-0.76] } \\
\text {-GAD (GADSS): } \\
\text { Diff -2.34 [-3.22 } \\
\text { to }-1.45] p<0.001 ; \\
\text { ES } 0.49[0.30 \text { to } 0.68]\end{array}$ \\
\hline
\end{tabular}


Table 1 Characteristics of randomized controlled trials comparing collaborative care for anxiety disorders with care as usual (Continued)

\begin{tabular}{|c|c|c|c|c|c|c|c|c|c|c|c|c|c|}
\hline 6 & $\begin{array}{l}\text { Oosterbaan } \\
\text { et al. 2013 } \\
\text { (Study 6) }\end{array}$ & $\begin{array}{l}\text { Clus-ter } \\
\mathrm{RCT}\end{array}$ & Referral & $\begin{array}{l}\mathrm{PD} / \\
\mathrm{AGO} / \\
\mathrm{GAD} / \\
\mathrm{SOP} / \\
\mathrm{SP} \\
\mathrm{MINI}\end{array}$ & $\begin{array}{l}\text { CC } \\
\text { VS } \\
\text { CAU }\end{array}$ & $\begin{array}{l}22 \\
\text { primary } \\
\text { care } \\
\text { practices } \\
(\mathrm{NL})\end{array}$ & $\begin{array}{l}\text { CC: } \\
28 \\
\text { CAU: } \\
27\end{array}$ & $\begin{array}{l}\text { Step 1) CBT } \\
\text { based guided } \\
\text { self-help with } \\
\text { antidepressant } \\
\text { medication for } \\
\text { moderate } \\
\text { disorder } \\
\text { Step 2) CBT and } \\
\text { medication in } \\
\text { specialty care }\end{array}$ & $\begin{array}{l}\text { PCP, CM, } \\
\text { psychiatrist, } \\
\text { CBT } \\
\text { therapist }\end{array}$ & $\begin{array}{l}\text { CAU by PCP, i.e. } \\
\text { medication, } \\
\text { counseling or referral } \\
\text { to mental health } \\
\text { professional }\end{array}$ & $\begin{array}{l}\text { CGI-I, CGI-S, } \\
\text { HRS-A } \\
4,8,12 \text { months }\end{array}$ & $\begin{array}{l}\text {-Improved } \\
\text { anxiety } \\
\text { outcomes at } \\
4 \text { months }\end{array}$ & $\begin{array}{l}\text {-Anxiety (HRS-A) } \\
\text { CC: M } 6.14 \text { SD } 5.26 \\
\text { CAU M: } 8.11 \text { SD } 7.83 \\
p=0.02 \\
\text { ES } 0.29[-0.29 \text { to } 0.87]\end{array}$ \\
\hline & $\begin{array}{l}\text { Muntingh } \\
\text { et al. 2014 } \\
\text { (Study 7) }\end{array}$ & $\begin{array}{l}\text { Clus-ter } \\
\text { RCT }\end{array}$ & $\begin{array}{l}\text { Referral } \\
\text { Screening } \\
\text { (PHQ) }\end{array}$ & $\begin{array}{l}\mathrm{PD} / \\
\mathrm{GAD} \\
\text { MINI }\end{array}$ & $\begin{array}{l}\text { CC } \\
\text { VS } \\
\text { CAU }\end{array}$ & $\begin{array}{l}43 \\
\text { primary } \\
\text { care } \\
\text { practices } \\
\text { (NL) }\end{array}$ & $\begin{array}{l}\text { CC: } \\
114 \\
\text { CAU: } \\
66\end{array}$ & $\begin{array}{l}\text { Step 1) CBT } \\
\text { based guided } \\
\text { self-help } \\
\text { Step 2) CBT } \\
\text { Step 3) } \\
\text { antidepressant } \\
\text { medication }\end{array}$ & $\begin{array}{l}\text { PCP, CM, } \\
\text { psychiatrist, } \\
\text { CBT } \\
\text { therapist }\end{array}$ & $\begin{array}{l}\text { CAU by PCP, i.e. } \\
\text { medication, } \\
\text { counseling or referral } \\
\text { to mental health } \\
\text { professional (including } \\
\text { CM randomized to } \\
\text { CAU) }\end{array}$ & BAI & $\begin{array}{l}\text {-Improved } \\
\text { anxiety } \\
\text { outcome at all } \\
\text { time points } \\
\text {-Improved } \\
\text { panic outcome } \\
\text { at all time } \\
\text { points } \\
\text {-No sign. } \\
\text { improvement } \\
\text { in GAD } \\
\text { outcomes }\end{array}$ & $\begin{array}{l}\text {-Anxiety (BAl) } \\
\text { Diff }-6.84[-10.13 \\
\text { to }-3.55] \\
p<0.001 ; \\
\text { ES 0.73 [0.37-1.09] } \\
\text { - PD (BAl): } \\
\text { Diff }-9.29[-12.99 \\
\text { to }-5.59] \\
\text { ES } 1.03 \text { [0.60 to } 1.46] \\
- \text { GAD (BAI): } \\
\text { Diff }-1.13[-7.33 \text { to } 5.08] \\
p=0.72 \\
\text { ES } 0.13[-0.56-0.81]\end{array}$ \\
\hline
\end{tabular}

and $\quad-$ SOP (SPIN): Diff -5.71

12 months [ $\quad-10.74$ to -0.68$] p=0.08$

$\begin{array}{ll}12 \text { months } & {[-10.74 \text { to }-0.68] p=0.08} \\ \text {-No sign. } & \text { ES } 0.43 \text { [0.05 to } 0.81]\end{array}$

. No sign.

improvement - PTSD (PCL-C): Dif -7.7

in PTSD $\quad[-17.55$ to 2.15$] p=0.49$

outcomes ES $0.45[-0.12$ to 1.02$]$

Oosterbaan Clus-ter Referral

$\mathrm{PD} / \mathrm{CC} 22$

VS primary 28 based guided

GAD/ CAU care CAU: self-help with

antidepressan

Step 2) CBT and

medication in

et al. 2014 RCT Screening

D/ CC 43

CC: $\quad$ Step 1) CBT

psychiatr

practices 66 Step 2) CBT

Step 3)

antidepressant

professional (including

CM randomized to

-No sign. $\quad$ ES $1.03[0.60$ to 1.46$]$

improvement - GAD (BAI):

Abbreviations: $A D$ anxiety disorder, $A S I$ anxiety sensitivity index, BAI Beck Anxiety Inventory, CAU care as usual, $C B T$ cognitive behavioral therapy, $C C$ collaborative care, $C I$ confidence interval, $C I D I$ Composite

International Diagnostic Interview, CM care manager, ES effect size, GAD generalized anxiety disorder, GADSS Generalized Anxiety Disorder Severity Scale, GER Germany, HRS-A Hamilton Rating Scale for Anxiety, ITI

intention to treat, MINI Mini-International Neuropsychiatric Interview, NL Netherlands, PD Panic disorder, PCL-C PTSD Checklist-Civilian Version, PCP primary care physician, PDSS panic disorder severity scale, PHQ Patient Health Questionnaire, PRIME-MD Primary Care Evaluation of Mental Disorders, PTSD post traumatic stress disorder, SIGH-A Hamilton Anxiety Rating Scale, SOP social phobia, SP specific phobia, SPIN Social Phobia Inventory, US United States 


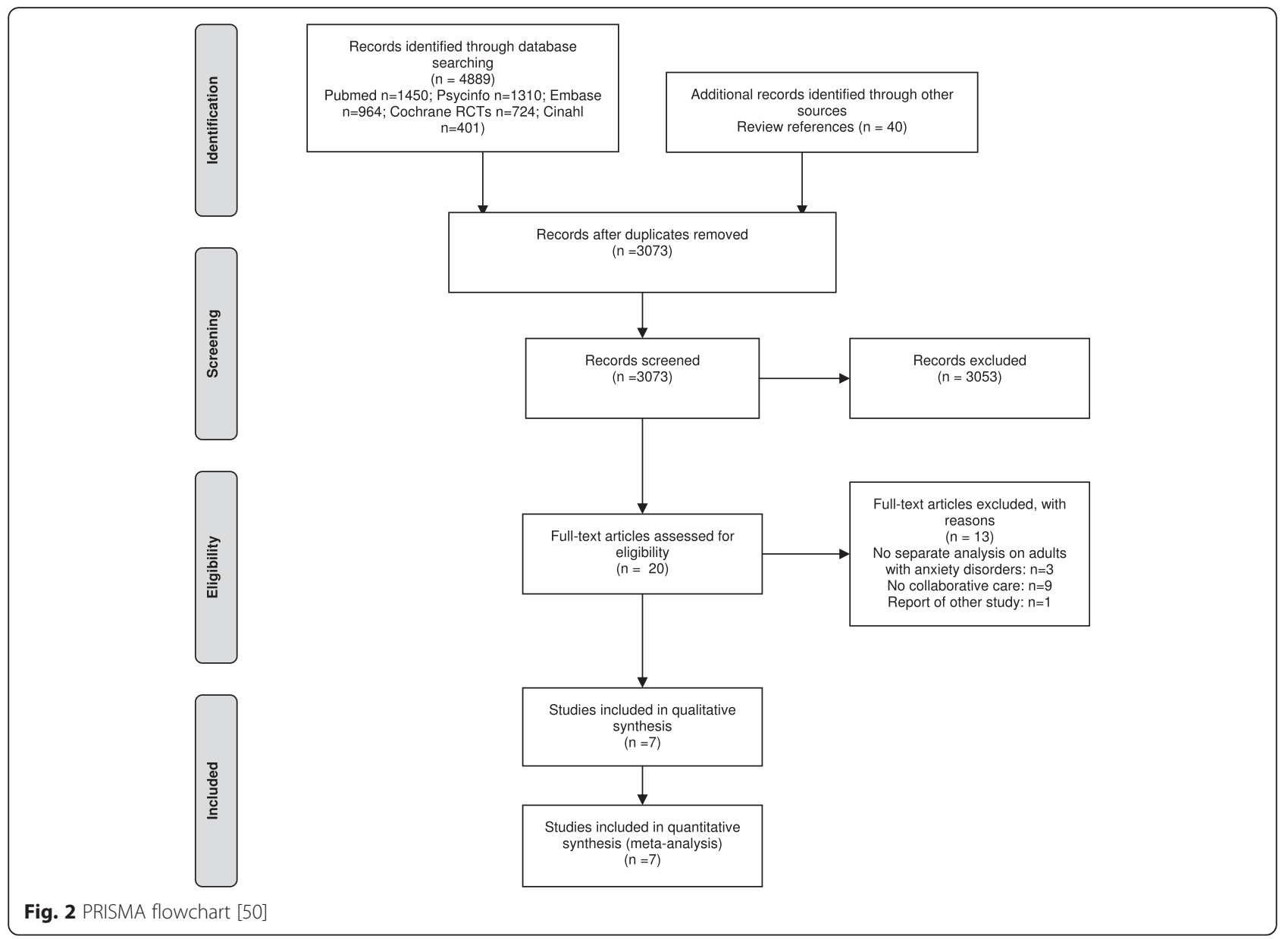

$1-3,5$ ), one in Germany (study 4) and two in the Netherlands (study 6 and 7). All studies reported that their study protocol was approved by the relevant medical ethical committee (Study 4, 6 and 7) or the institutional review board (study 1-3, and 5). Two studies exclusively included patients with panic disorder (studies 1 and 2), two studies (studies 3 and 7) included patients with panic disorder and/or generalized anxiety, and three studies (studies 4-6) included multiple anxiety disorders. Comorbid depression was allowed in all studies and was reported in five studies (Study $1-3,5,7)$, with prevalence rates ranging from $31 \%$ (Study 7) to $64 \%$ (Study 5). With one exception, studies used a structured interview to classify the anxiety disorder at baseline (CIDI, PRIME-MD). König and colleagues (study 4) determined the presence of an anxiety disorder by a cut-off score on the Patient Health Questionnaire. To recruit participants, two studies used screening only (studies 3 and 4), two studies (studies 5 and 6) used referral of primary care physicians, while three studies used both methods (studies 1,2,7).

\section{Collaborative care and comparison interventions}

The content of collaborative care interventions varied considerably between studies. In all studies, a primary care physician and a psychiatrist were involved, while in five studies a care manager was introduced as well (studies 2,3,5-7). One study used only medication management by a psychiatrist (study 1 ) and one study evaluated counselling with CBT elements by a trained primary care physician (study 4). The other studies used more comprehensive programs with a choice between, or combination of, antidepressant medication and CBT. Two studies (studies 6 and 7) used a stepped care program. Table 2 describes the characteristics of collaborative care in the included studies.

All studies compared the collaborative care intervention to usual care coordinated by the primary care physician. Table 3 gives an overview of the percentage of patients receiving pharmacotherapy, appropriate pharmacotherapy, counseling, CBT, and (specialized) mental healthcare as reported in the included studies. Pharmacotherapy was the most frequent treatment method reported in usual care. 
Table 2 Characteristics of collaborative care interventions for anxiety disorders

\begin{tabular}{|c|c|c|c|c|c|c|c|c|}
\hline $\begin{array}{l}\text { Study } \\
\text { no. }\end{array}$ & $\begin{array}{l}\text { Study } \\
\text { (first author, } \\
\text { year) }\end{array}$ & $\begin{array}{l}\text { Professionals } \\
\text { involved }\end{array}$ & Professional training & Interventions used & $\begin{array}{l}\text { No. contacts } \\
\text { with } \\
\text { professionals }\end{array}$ & $\begin{array}{l}\text { Collaboration } \\
\text { between } \\
\text { professionals }\end{array}$ & Monitoring & $\begin{array}{l}\text { Follow-up / } \\
\text { relapse } \\
\text { prevention }\end{array}$ \\
\hline 1 & $\begin{array}{l}\text { Roy-Byrne } \\
\text { et al. } 2001\end{array}$ & $\begin{array}{l}\text { PCP } \\
\text { Psychiatrist }\end{array}$ & $\begin{array}{l}\text { PCP: 1-h didactic, } \\
\text { medication } \\
\text { algorithm }\end{array}$ & $\begin{array}{l}\text { Medication management } \\
\text { (paroxetine) and encouragement } \\
\text { of adherence and exposure by } \\
\text { psychiatrist } \\
\text { Educational patient video }\end{array}$ & $\begin{array}{l}2 \text { visits and } 2 \\
\text { phone calls by } \\
\text { psychiatrist }\end{array}$ & $\begin{array}{l}\text { The PCP received a typed } \\
\text { consultation note after each } \\
\text { psychiatric visit. }\end{array}$ & $\begin{array}{l}\text { No information } \\
\text { provided }\end{array}$ & $\begin{array}{l}5 \text { follow-up calls by } \\
\text { psychiatrist }\end{array}$ \\
\hline 2 & $\begin{array}{l}\text { Roy-Byrne } \\
\text { et al. } 2005\end{array}$ & $\begin{array}{l}\text { PCP, } \\
\text { Psychiatrist } \\
\text { CM }\end{array}$ & $\begin{array}{l}\text { PCP: 1-h didactic } \\
\text { on, medication algorithm } \\
\text { CM: treatment protocol, } \\
\text { six videotapes, } 1 \text { day long } \\
\text { workshop in care } \\
\text { management and CBT }\end{array}$ & $\begin{array}{l}\text { Face-to-face CBT } \\
\text { Antidepressant medication } \\
\text { according to algorithm by PCP } \\
\text { Educational video and workbook }\end{array}$ & $\begin{array}{l}6 \text { sessions by } \\
\mathrm{CM}\end{array}$ & $\begin{array}{l}\text { Weekly caseload supervision of } \\
\text { CM by psychiatrist } \\
\text { 2-way communication of CM } \\
\text { and PCP by telephone, fax, and } \\
\text { e-mail. } \\
\text { Recommendations as needed } \\
\text { from a consulting psychiatrist } \\
\text { to the PCP via the CM }\end{array}$ & $\begin{array}{l}\text { No information } \\
\text { provided }\end{array}$ & $\begin{array}{l}\text { Intended were } 6 \\
\text { telephone follow-up } \\
\text { contacts during } \\
10 \text { months after the } \\
\text { active treatment } \\
\text { phase by CM }\end{array}$ \\
\hline 3 & $\begin{array}{l}\text { Rollman et } \\
\text { al. } 2005\end{array}$ & $\begin{array}{l}\text { PCP, } \\
\text { Psychiatrist } \\
\text { CM }\end{array}$ & $\begin{array}{l}\text { PCP: 1-h conference and in } \\
\text { dividual meeting of study } \\
\text { investigators with PCPs, } \\
\text { medication algorithm } \\
\text { CM: study protocol and } \\
\text { self-management workbooks, } \\
\text { attending lectures at the } \\
\text { University }\end{array}$ & $\begin{array}{l}\text { CBT based guided self-help } \\
\text { Antidepressant medication } \\
\text { according to algorithm by PCP } \\
\text { Referral to a community mental } \\
\text { health specialist }\end{array}$ & $\begin{array}{l}7 \text { telephone } \\
\text { contacts by } \\
\text { CM }\end{array}$ & $\begin{array}{l}\text { Weekly caseload supervision of } \\
\text { CM by psychiatrist } \\
\text { Advice from the psychiatrist to } \\
\text { the PCP and patient via CM } \\
\text { Communication facilitated } \\
\text { through an ambulatory EMR } \\
\text { system }\end{array}$ & $\begin{array}{l}\text { Monitoring by } \\
\text { CM with PDSS / } \\
\text { GADSS }\end{array}$ & $\begin{array}{l}\text { Telephone contacts } \\
\text { every } 1-3 \text { months } \\
\text { after the acute phase } \\
\text { to monitor symptoms }\end{array}$ \\
\hline 4 & $\begin{array}{l}\text { König et } \\
\text { al.2009 }\end{array}$ & $\begin{array}{l}\text { PCP, } \\
\text { Psychiatrist/ } \\
\text { Clinical } \\
\text { psychologist }\end{array}$ & $\begin{array}{l}\text { PCP: } 10 \mathrm{~h} \text { training and two } \\
\text { additional sessions on } \\
\text { counseling skills and CBT }\end{array}$ & $\begin{array}{l}\text { Counseling by PCP, including } \\
\text { CBT techniques }\end{array}$ & $\begin{array}{l}\text { No information } \\
\text { provided }\end{array}$ & $\begin{array}{l}\text { As needed consultation } \\
\text { by psychiatrist/clinical } \\
\text { psychologist at PCPs' } \\
\text { practices }\end{array}$ & $\begin{array}{l}\text { No information } \\
\text { provided }\end{array}$ & $\begin{array}{l}\text { No information } \\
\text { provided }\end{array}$ \\
\hline 5 & $\begin{array}{l}\text { Roy-Byrne } \\
\text { et al. } 2010\end{array}$ & $\begin{array}{l}\text { PCP, } \\
\text { Psychiatrist } \\
\text { CM }\end{array}$ & $\begin{array}{l}\text { PCP: single-session training, } \\
\text { medication algorithm } \\
\text { CM: treatment protocol, } 6 \\
\text { half days of didactics in care } \\
\text { management and CBT }\end{array}$ & $\begin{array}{l}\text { Face-to-face CBT by CM } \\
\text { supported by computer program } \\
\text { Antidepressant medication } \\
\text { according to algorithm by PCP }\end{array}$ & $\begin{array}{l}6-8 \text { sessions } \\
\text { by } \mathrm{CM}\end{array}$ & $\begin{array}{l}\text { Weekly caseload supervision } \\
\text { of CM by psychiatrist/psychologist } \\
\text { "Regular" interaction between } \\
\text { PCP and CM in person and by } \\
\text { telephone } \\
\text { As needed consultation of PCP } \\
\text { by psychiatrist } \\
\text { Communication facilitated } \\
\text { through a webbased } \\
\text { monitoring system }\end{array}$ & $\begin{array}{l}\text { Monitoring with } \\
\text { OASIS by CM }\end{array}$ & $\begin{array}{l}\text { Monthly follow-up } \\
\text { telephone calls by } \\
\text { CM }\end{array}$ \\
\hline 6 & $\begin{array}{l}\text { Oosterbaan } \\
\text { et al. } 2013\end{array}$ & $\begin{array}{l}\text { PCP } \\
\text { Psychiatrist/ } \\
\text { CBT specialist } \\
\text { CM }\end{array}$ & $\begin{array}{l}\text { PCP: one educational session, } \\
\text { medication algorithm } \\
\text { CM: treatment protocol, } \\
\text { 2-day training session in } \\
\text { basic CBT strategies }\end{array}$ & $\begin{array}{l}\text { Stepped care (according } \\
\text { to severity): } \\
\text { 1. CBT based guided self-help } \\
\text { with support by CM (face-to- } \\
\text { face) with antidepressant } \\
\text { medication according to } \\
\text { algorithm by PCP for patients } \\
\text { with a moderate disorder } \\
\text { 2. CBT and antidepressants in } \\
\text { specialised mental health service }\end{array}$ & $\begin{array}{l}\text { Step 1: } 5 \text { sessions } \\
\text { by CM } \\
\text { Step 2: No } \\
\text { information } \\
\text { provided }\end{array}$ & $\begin{array}{l}\text { 2-weekly supervision of CM by } \\
\text { CBT specialist } \\
\text { As needed consultation of PCP } \\
\text { by psychiatrist }\end{array}$ & $\begin{array}{l}\text { Monitoring with } \\
\text { CGl by CM }\end{array}$ & $\begin{array}{l}\text { No information } \\
\text { provided }\end{array}$ \\
\hline
\end{tabular}


Table 2 Characteristics of collaborative care interventions for anxiety disorders (Continued)

\begin{tabular}{|c|c|c|c|c|c|c|c|c|}
\hline 7 & $\begin{array}{l}\text { Muntingh } \\
\text { et al. } 2014\end{array}$ & $\begin{array}{l}\text { PCP } \\
\text { Psychiatrist/ } \\
\text { CBT specialist } \\
\text { CM }\end{array}$ & $\begin{array}{l}\text { PCP: } 3 \text { h workshop, } \\
\text { medication algorithm } \\
\text { CM: treatment protocol, } \\
\text { 3-day workshop in care } \\
\text { management and CBT } \\
\text { strategies }\end{array}$ & $\begin{array}{l}\text { Stepped care: } \\
\text { 1. CBT based guided } \\
\text { self-help with support } \\
\text { by CM (face-to-face) } \\
\text { 2. CBT by CM } \\
\text { 3. Antidepressant medication } \\
\text { according to algorithm } \\
\text { by PCP }\end{array}$ & $\begin{array}{l}\text { Step 1: } 5 \text { sessions } \\
\text { by CM } \\
\text { Step 2: } 6 \text { sessions } \\
\text { by CM }\end{array}$ & $\begin{array}{l}\text { Intended was 3-weekly } \\
\text { supervision of CM by } \\
\text { psychiatrist/ } \\
\text { CBT specialist } \\
\text { GP and CM were "instructed } \\
\text { to frequently discuss } \\
\text { treatment progress" } \\
\text { As needed consultation } \\
\text { of PCP by psychiatrist }\end{array}$ & $\begin{array}{l}\text { Monitoring with } \\
\text { BAl by CM }\end{array}$ & $\begin{array}{l}\text { Monthly follow-up } \\
\text { telephone calls } \\
\text { by CM }\end{array}$ \\
\hline
\end{tabular}

Abbreviations: $C B T$ cognitive behavioral therapy, $C M$ care manager, $P C P$ primary care phyisican 
Table 3 Care received in the collaborative care and care as usual conditions ( $N=7)$

\begin{tabular}{|c|c|c|c|c|c|c|c|c|c|c|}
\hline \multirow{2}{*}{$\begin{array}{l}\text { Content of care* } \\
\text { Study }\end{array}$} & \multicolumn{2}{|c|}{ Pharmaco-therapy (\%) } & \multicolumn{2}{|c|}{$\begin{array}{l}\text { Approriate pharmaco- } \\
\text { therapy (\%) }\end{array}$} & \multicolumn{2}{|c|}{ Counseling (\%) } & \multicolumn{2}{|c|}{ CBT (\%) } & \multicolumn{2}{|c|}{$\begin{array}{l}\text { Referral to mental } \\
\text { health professional (\%) }\end{array}$} \\
\hline & CC & CAU & $\mathrm{CC}$ & CAU & CC & CAU & CC & CAU & CC & CAU \\
\hline Roy-Byrne et al. 2001* & $77 \%^{a}$ & $48 \%^{a}$ & $47 \%{ }^{b}$ & $33 \%{ }^{b}$ & NA & NA & NA & NA & NA & $25 \%$ \\
\hline Roy-Byrne et al. 2005 & $54 \%^{c}$ & $52 \%^{c}$ & $41 \%{ }^{b}$ & $39 \%$ & $70 \%$ & $34 \%$ & $63 \%^{d}$ & $14 \%^{d}$ & NA & NA \\
\hline Rollman et al. 2005 & $77 \%{ }^{e}$ & $66 \%{ }^{e}$ & NA & NA & $79 \%^{f}$ & NA & $66 \%^{9}$ & NA & $18 \%$ & $26 \%$ \\
\hline König et al. 2009 & NA & NA & NA & NA & NA & NA & NA & NA & $33 \%$ & $33 \%$ \\
\hline Roy-Byrne et al. 2010 & $70 \%{ }^{h}$ & $68 \%^{h}$ & $46 \%^{i}$ & $42 \%$ & $88 \%$ & $51 \%$ & $82 \%$ & $34 \%$ & NA & NA \\
\hline Oosterbaan et al. 2013* & $45 \%$ & $33 \%$ & NA & NA & NA & NA & $75 \%{ }^{9}$ & NA & NA & NA \\
\hline Muntingh et al. 2014 & $21 \%^{e}$ & $35 \%{ }^{e}$ & NA & NA & $92 \%$ & $12 \%$ & $78 \%{ }^{9}$ & NA & $11 \%$ & $21 \%$ \\
\hline
\end{tabular}

*Highest \% of patients that have received a form of care at any follow-up measurement

${ }^{a}$ Appropriate type of medication

${ }^{\mathrm{b}}$ Adequate dose and duration of medication

c Any antipanic pharmacotherapy

$d_{3}$ or more sessions counseling plus at least 4 of 7 CBT techniques

eSSRI/SNRI pharmacotherapy

$\mathrm{f}_{3}$ or more telephone contacts with $\mathrm{CM}$

${ }^{9} 3$ or more (telephone) contacts with $\mathrm{CM}$ about CBT workbook

${ }^{\mathrm{h}}$ Any psychotropic medication

'Appropriate type, dose and duration

Counseling with at least 3 CBT elements

$N A=$ Not Available

\section{Outcome measures}

All studies included a continuous outcome scale to measure anxiety. Study 2 used a dichotomous outcome (remission and response) as primary outcome measure. Five studies reported separately about panic disorder outcomes (studies 1-3,5,7). The length of follow-up varied from 9 months (study 4) to 18 months (study 5). Two studies (studies 4 and 7) used patient self-report to assess the outcome, while in the other five studies a (blinded) research assistant administered the outcome measures by telephone.

\section{Risk of bias within studies}

The overall quality of included studies was moderate to high (see Table 4). For study 1, six out of eight criteria were rated as unclear; in the other studies four to six criteria were rated as low risk of bias. The most prevalent potential source of bias was the inability to blind patients and professionals for treatment allocation, as is common in psychotherapy research [38]. Studies 6 and 7 used cluster randomization with subsequent referral by the primary care physician, which may have induced selection bias. Study 1 did not provide the statistics of an insignificant result on the Panic Disorder Severity Scale (PDSS). Of four studies (studies 2,3,5, 7), a published study protocol was retrieved [39-42].

\section{Results of individual studies}

The results of the individual studies at follow-up closest to 12 months are reported in Table 1 . All studies except study 4 reported a significantly greater effect of the collaborative care intervention compared to care as usual. The meta-analysis (Fig. 3), pooling the data of all seven studies, yielded an SMD of 0.35 (95\% CI 0.14 to $0.56, p=0.001$ ), indicating that collaborative care leads to a significantly greater reduction in anxiety symptoms, with a small effect size after 12 months. The $Q$-value was 21.73 $(\mathrm{df}=6, p=0.001)$, indicating significant dispersion across

Table 4 Risk of bias in 7 randomized controlled trials comparing collaborative care for adult patients with anxiety disorders to usual primary care

\begin{tabular}{|c|c|c|c|c|c|c|c|c|}
\hline & $\begin{array}{l}\text { Adequate } \\
\text { Sequence } \\
\text { Generation? }\end{array}$ & $\begin{array}{l}\text { Allocation } \\
\text { concealed? }\end{array}$ & $\begin{array}{l}\text { Patients } \\
\text { blinded? }\end{array}$ & $\begin{array}{l}\text { Professionals } \\
\text { blinded? }\end{array}$ & $\begin{array}{l}\text { Outcome } \\
\text { assessors } \\
\text { blinded? }\end{array}$ & $\begin{array}{l}\text { Incomplete } \\
\text { outcome } \\
\text { data addressed? }\end{array}$ & $\begin{array}{l}\text { Free of selective } \\
\text { reporting? }\end{array}$ & $\begin{array}{l}\text { Free of } \\
\text { other bias }\end{array}$ \\
\hline Roy-Byrne et al. 2001 & + & $?$ & $?$ & $?$ & + & $?$ & $?$ & $?$ \\
\hline Roy-Byrne et al. 2005 & $?$ & + & - & - & + & + & $?$ & + \\
\hline Rollman et al. 2005 & + & + & - & - & + & + & $?$ & + \\
\hline König et al. 2009 & + & + & - & - & $?$ & + & $?$ & + \\
\hline Roy-Byrne et al. 2010 & + & + & $?$ & - & + & + & + & + \\
\hline Oosterbaan et al. 2013 & + & + & - & - & + & + & + & + \\
\hline Muntingh et al. 2014 & + & + & - & - & + & + & + & + \\
\hline
\end{tabular}




\begin{tabular}{|c|c|c|c|c|c|c|c|c|c|}
\hline \multirow[t]{2}{*}{ Study name } & \multirow[t]{2}{*}{ Subgroup within study } & \multirow[t]{2}{*}{ Outcome } & \multicolumn{7}{|c|}{$\underline{\text { Statistics for each study }}$} \\
\hline & & & $\begin{array}{l}\text { Std diff } \\
\text { in means }\end{array}$ & $\begin{array}{l}\text { Standard } \\
\text { error }\end{array}$ & Variance & $\begin{array}{c}\text { Lower } \\
\text { limit }\end{array}$ & $\begin{array}{l}\text { Upper } \\
\text { limit }\end{array}$ & Z-Value & $\mathrm{p}$-Value \\
\hline Roy-Byrne et al. 2001 & Anxiety & ASI & 0.449 & 0.212 & 0.045 & 0.033 & 0.865 & 2.114 & 0.035 \\
\hline Roy-Byrne et al. 2005 & Anxiety & ASI & 0.481 & 0.152 & 0.023 & 0.184 & 0.778 & 3.170 & 0.002 \\
\hline Rollman et al. 2005 & Anxiety & SIGH-A & 0.430 & 0.171 & 0.029 & 0.095 & 0.765 & 2.514 & 0.012 \\
\hline Konig et al. 2009 & Anxiety & BAI & -0.129 & 0.117 & 0.014 & -0.358 & 0.099 & -1.108 & 0.268 \\
\hline Roy-Byrne et al. 2010 & Anxiety & BSI & 0.331 & 0.071 & 0.005 & 0.192 & 0.469 & 4.683 & 0.000 \\
\hline Oosterbaan et al. 2013 & Anxiety & HRS-A & 0.290 & 0.298 & 0.089 & -0.293 & 0.874 & 0.976 & 0.329 \\
\hline Muntingh et al. 2014 & Anxiety & BAI & 0.730 & 0.183 & 0.033 & 0.371 & 1.089 & 3.989 & 0.000 \\
\hline & & & 0.352 & 0.108 & 0.012 & 0.140 & 0.563 & 3.260 & 0.001 \\
\hline
\end{tabular}

Favours care as usual Std diff in means and $95 \% \mathrm{Cl}$

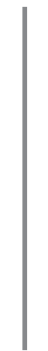

100

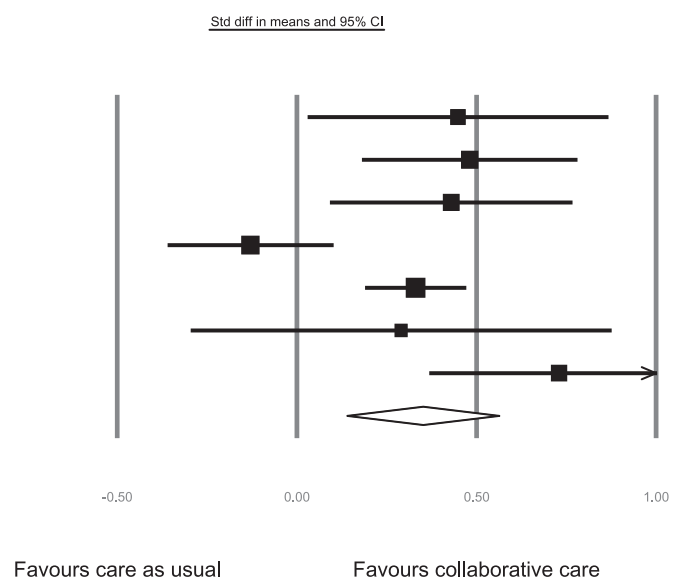

Fig. 3 Meta-analysis for the effect of collaborative care vs. care as usual on continuous anxiety scales at 12 months follow-up

studies. The $I^{2}$ was $72 \%$, which indicates that a high proportion of the total variation may be attributed to true heterogeneity between studies. A sensitivity analysis excluding Study 4 led to a considerable decrease in heterogeneity $\left(Q=4.72, \mathrm{df}=5, p=0.34 ; I^{2}=0 \%\right)$ and a higher SMD (SMD $=0.40,95 \%$ CI 0.30 to $0.51, p<0.001$ ).

\section{Risk of bias across studies}

The funnel plot showed an indication (not significant) for publication bias for the analysis on all anxiety disorders (Egger's test, two-tailed $p=0.57$ ). According to the trim and fill method [32], the SMD should be adjusted from 0.35 to 0.25 (95\% CI 0.16 to 0.34 ). This asymmetry may indicate that the effect of collaborative care for anxiety disorders is slightly overestimated due to publication bias, although asymmetry may also be attributed to true heterogeneity between studies [43]. Indeed, heterogeneity was high $\left(I^{2}=72 \%\right)$, which may be attributed to differences between the three European studies $\left(I^{2}=78 \%\right.$ in European studies vs. $I^{2}=0 \%$ in US studies). For the subgroup analysis on panic disorder, no indication was found for a publication bias (Egger's test, two-tailed $p=0.29$ ). Furthermore, we ran a sensitivity analysis excluding the lowest quality study (Study 1). This had no significant effect on the analysis on anxiety disorders $\left(\mathrm{SMD}_{\text {adjusted }} 0.34,95 \% \mathrm{CI} 0.10-0.58\right)$ or panic disorder ( $\mathrm{SMD}_{\text {adjusted }} 0.60,95 \% \mathrm{CI} 0.38$ to 0.83 ).

\section{Additional analysis: disorder-specific impact of collaborative care}

A predefined subgroup analysis was performed for patients with panic disorder. Outcomes on the Panic Disorder Severity Scale (PDSS) were used when reported. For the two studies $(2,7)$ that did not report the PDSS, the primary outcome measure was used in the metaanalysis. Because Roy-Byrne and colleagues [33] reported an insignificant result on the PDSS, but were not able to provide the statistics necessary for calculating the effect size, we used the ASI score. The combined effect size of the four studies comparing collaborative care to care as usual in patients with panic disorder was 0.59 (95\% CI 0.41 to $0.78, p<0.001$ ), which may be interpreted as a moderate effect size (Fig. 4).

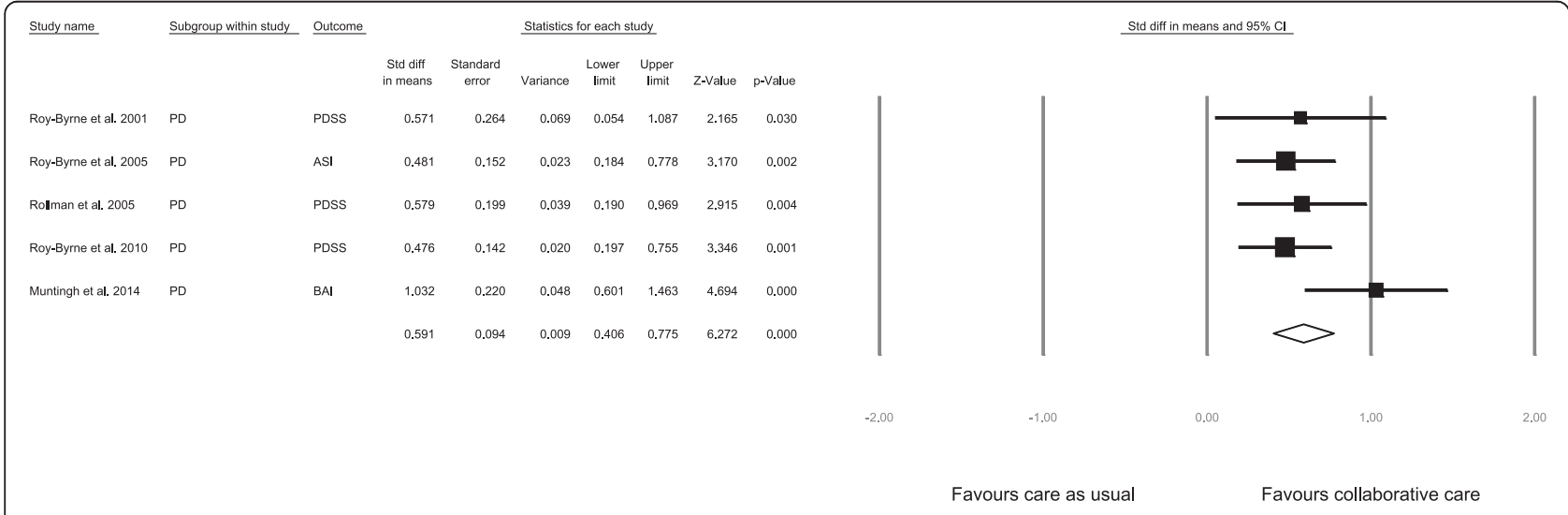

Fig. 4 Subgroup-analysis for the effect of collaborative care vs. care as usual at 12 months follow-up for patients with a panic disorder 
Concerning anxiety disorders other than panic disorder, studies 3 and 7 reported no significant effect for patients with generalized anxiety disorder only (Table 1). Study 5 reported a significant difference between collaborative care and care as usual on the Brief Symptom Inventory (BSI) for panic disorder, generalized anxiety disorder, and social phobia, but not for posttraumatic stress disorder (PTSD). In a subsequent report on this study [44], disorder-specific data on specific scales were reported, with similar results (Table 1).

\section{Additional analysis: variables related to outcome}

Additional analyses were performed to assess the impact on outcome of variables related to study procedure and content of the intervention (see [25]; Table 5). The analysis revealed that studies performed in the United States were more homogeneous $\left(I^{2}=0 \%\right.$ vs. $\left.87 \%\right)$ and on average yielded a significantly greater effect size than studies performed in European countries (SMD 0.37 vs. 0.29, $p$ $=0.03$ ). Furthermore, the five studies that included a care manager had a significantly higher effect size (SMD 0.42 vs. $0.13, p=0.001$ ) than those without. Lastly, the two studies using stepped collaborative care (both from the Netherlands) yielded a greater effect size than studies that did not use stepped care (SMD 0.57 vs. $0.29, p=$ 0.04). However, these analyses need to be interpreted with caution due to the small number of studies and the considerable heterogeneity between European studies.

\section{Discussion}

\section{Summary of evidence}

The results of this meta-analysis indicate that collaborative care is more effective than usual primary care at twelve months follow-up, with a small effect size for all anxiety disorders combined (SDM 0.35) and a moderate effect size for patients with panic disorder (SMD 0.59). The quality of studies was moderate to high. There were indications for considerable heterogeneity between European studies in contrast to studies performed in the United States.

\section{Strengths and limitations}

A strength of this study is that we conducted this review using PRISMA criteria [26]. Furthermore, we were able to

Table 5 Meta-analysis with between-study subgroup analyses of variables related to study procedures and content of collaborative care

\begin{tabular}{|c|c|c|c|c|c|c|}
\hline Covariate & Number & SDM & $95 \% \mathrm{Cl}$ & Q & $1^{2}$ & $P$ \\
\hline All studies & 7 & 0.35 & $0.14-0.56$ & 21.73 & 72.39 & \\
\hline Country & & & & & & $0.031^{*}$ \\
\hline US & 4 & 0.37 & $0.26-0.49$ & 1.10 & 0.00 & \\
\hline European & 3 & 0.29 & $-0.31-0.89$ & 15.98 & 87.50 & \\
\hline Randomization procedure & & & & & & $0.031^{*}$ \\
\hline Patient randomization & 4 & 0.37 & $0.26-0.49$ & 1.10 & 0.00 & \\
\hline Cluster randomization & 3 & 0.29 & $-0.31-0.89$ & 15.98 & 87.50 & \\
\hline Recruitment method & & & & & & 0.65 \\
\hline Referral by professional & 2 & 0.33 & $0.19-0.47$ & 0.02 & 0.00 & \\
\hline Systematic identification (or both) & 5 & 0.38 & $0.05-0.71$ & 21.51 & 81.41 & \\
\hline Care manager & & & & & & $0.001^{*}$ \\
\hline Care manager & 5 & 0.42 & $0.29-0.55$ & 4.66 & 14.24 & \\
\hline No care manager & 2 & 0.13 & $-0.43-0.70$ & 5.69 & 82.43 & \\
\hline Intervention content & & & & & & 0.82 \\
\hline Psychological intervention (CBT) with/without medication management & 5 & 0.42 & $0.29-0.55$ & 4.66 & 14.24 & \\
\hline Medication management alone & 1 & 0.45 & $0.03-0.87$ & - & - & \\
\hline Not applicable & 1 & - & - & - & - & \\
\hline Stepped care & & & & & & $0.041^{*}$ \\
\hline Stepped care & 2 & 0.57 & $0.06-0.53$ & 1.58 & 36.85 & \\
\hline No stepped care & 5 & 0.29 & $0.16-0.99$ & 15.97 & 74.95 & \\
\hline Supervision frequency specialist-care manager & & & & & & 0.056 \\
\hline Ad hoc & 1 & 0.73 & $0.37-1.09$ & - & - & \\
\hline Scheduled (i.e. at least 2-weekly) & 4 & 0.36 & $0.25-0.48$ & 1.03 & 0.00 & \\
\hline Not applicable & 2 & - & - & - & - & \\
\hline
\end{tabular}


examine the long-term effects of collaborative care, which is often problematic in reviewing effectiveness studies on anxiety disorders in primary care [45]. However, there are several limitations in this systematic review and metaanalysis that need consideration.

We identified only seven studies that met our inclusion criteria. As our search was limited to published articles, we probably have missed (unpublished) RCTs, which generally report lower effect sizes [46]. For example, we omitted a study by Rollman and colleagues [47], including 250 patients with generalized anxiety disorder or panic disorder, because their results remain unpublished. They reported an effect size of 0.30 in a conference abstract, which is comparable to the overall effect size found in our meta-analysis.

Furthermore, some methodological issues should be mentioned. One study [33] did not report the statistics for the insignificant effect on the disorder-specific measure. However, a sensitivity analysis excluding this study did not alter the results. Also, two studies [21, 22] used referral as a recruitment method after cluster randomization, which may cause selection bias and inflated effect sizes [48]. Yet Muntingh and colleagues, who used both referral and screening to recruit patients, reported nearly equal effect sizes in a subgroup analysis on patients selected by screening [22]. Lastly, one may debate on the broad concept of collaborative care. The seven studies that fulfilled our inclusion criteria varied on several characteristics, such as the inclusion of a care manager, care manager supervision, provision of $\mathrm{CBT}$, and following a stepped care protocol. Common elements in all seven studies were the input of specialist mental health in primary care, collaboration between professionals and the provision of evidence based interventions. As we are coming closer to defining the most effective elements of collaborative care (such as the provision of psychological treatment by a care manager [25]), the concept of collaborative care can be refined in the future, leading to more homogeneous results.

\section{Comparison with the literature}

The results from our meta-analysis compare to those reported in previous meta-analyses on collaborative care for anxiety disorders $[19,20]$ and extend their results with the inclusion of recent European and collaborative stepped care studies. The SMD of 0.35 found in our meta-analysis is also similar to the SMD of 0.28 which was reported in two large meta-analyses about collaborative care for depressive disorders $[19,25]$.

Comorbid depression was prevalent in the included studies (31-64\%). Unfortunately, none of the studies reported on the effects of comorbid depression on outcome, while depression has been related to a poor outcome of treatment for anxiety disorders [49].
We found that studies performed in the US had a greater effect size compared to European studies, which is in contrast with previous findings [25]. The US studies were also considerably more homogeneous than the European studies, which may be related to similarities and differences in healthcare systems between the US and Europe [16]. The studies in the US were mainly conducted in large clinics (often university-affiliated) using specifically trained and employed care managers, which may have facilitated effective implementation of the intervention, while the European studies were performed in more diverse settings in rather small primary care practices with collaborating professionals who were only involved in collaborative care on a part-time basis [21]. However, the heterogeneity between the European studies may also be related by other study characteristics such as design and intervention details. In fact, the study of König and colleagues [36] was the only study to report a non-significant effect of the intervention, and excluding this study from the analysis led to a considerable decrease in heterogeneity. The absence of a significant effect of this study could be related to a) suboptimal implementation and b) the fact that they employed the most 'basic' package of collaborative care. Both careful implementation and the application of a comprehensive collaborative care program may thus be crucial to the effectiveness of collaborative care. Including the study of König and colleagues probably provides a conservative estimate of the effects of collaborative care for anxiety disorders. In any case, compared to psychological treatment alone for anxiety disorders in primary care [45], the results of collaborative care are promising.

\section{Implications for research}

Most importantly, more randomized controlled trials and, subsequently, individual patient data meta-analyses are warranted to better identify who can benefit from what aspect of the intervention. We found a somewhat greater effect size for patients with panic disorder than for all anxiety disorders combined. In fact, evidence for the effectiveness of collaborative care for generalized anxiety disorders based on three included studies was inconclusive [21, 35, 37], so future research should address the effectiveness of collaborative care for anxiety disorders other than panic disorder. Furthermore, as described above, successful implementation of the intervention seems essential for the effectiveness of collaborative care. Hence, implementation should also be a focus of research on collaborative care. Lastly, cost-effectiveness analyses are needed to increase our knowledge on the benefits of collaborative care.

\section{Conclusions}

This meta-analysis indicates that collaborative care is more effective than usual primary care for adult patients with 
common anxiety disorders (small effect size), and panic disorder in particular (moderate effect size). Since it is difficult to improve primary care for anxiety disorders, this is a promising result. More research is needed to increase diagnostic precision, disentangle elements that make collaborative care most effective, and to evaluate the effectiveness of collaborative care in different anxiety disorders.

\begin{abstract}
Abbreviations
AD; anxiety disorder, ASI; Anxiety Sensitivity Index, BAl; beck anxiety inventory, BSI; Brief Symptom Inventory, CAU; care as usual, CBT; cognitive behavioral therapy, CC; collaborative care, Cl; confidence interval, CIDl; Composite international diagnostic interview, CM; care manager, ES; effect size, GAD; generalized anxiety disorder, GADSS; generalized anxiety disorder severity scale, GER; Germany, HRS-A; Hamilton rating scale for anxiety, ITT; intention to treat, MINI; mini-international neuropsychiatric interview, NL; Netherlands, PCL-C; PTSD checklist-civilian version, PCP; primary care physician, PD; Panic disorder, PDSS; panic disorder severity scale, PHQ; patient health questionnaire, PRIME-MD; primary care evaluation of mental disorders, PTSD; posttraumatic stress disorder, $\mathrm{RCT}$; randomized controlled trial, SIGH-A; Hamilton anxiety rating scale, SMD; standardized mean difference, SOP; social phobia, SP; specific phobia, SPIN; social phobia inventory, US; United States.
\end{abstract}

\section{Acknowledgements}

We thank librarian Caroline Planting for her assistance on the design and the performance of the literature search.

\section{Funding}

No funding was acquired.

\section{Availability of data and materials}

All relevant data are available in the manuscript or in the individual study reports.

\section{Authors' contributions}

AM participated in the literature search, screened titles and abstracts of retrieved studies, assessed full-text articles, extracted outcome data and other relevant characteristics of the studies, assessed risk of bias, performed the meta-analyses, and drafted the manuscript. CFC screened titles and abstracts of retrieved studies, assessed full-text articles, extracted outcome data, acted as the third reviewer when there was disagreement on the risk of bias, and supervised the performance of the meta-analyses. HvM and TVB assessed full-text articles and risk of bias, and co-authored the manuscript. PS co-authored the manuscript. All authors read and approved the final manuscript

\section{Authors' information}

Not provided.

\section{Competing interests}

The authors declare that they have no competing interests.

\section{Consent for publication}

Not applicable.

\section{Ethics approval and consent to participate}

Not applicable.

\footnotetext{
Author details

'Department of Psychiatry, VU University Medical Center / GGZ inGeest, A.J. Ernststraat 1187, Amsterdam $1081 \mathrm{HL}$, The Netherlands. ${ }^{2}$ Faculty of Social Sciences, Tranzo department, Tilburg University, PO Box 90153, Tilburg 5000 LE, The Netherlands. ${ }^{3}$ Top Clinical Centre for Body, Mind and Health, GGZ Breburg, Lage Witsiebaan 4, Tilburg 5042 DA, The Netherlands. ${ }^{4}$ Centre for Primary Care, Institute for Population Health, University of Manchester, Manchester, UK. ${ }^{5}$ Department of General Practice and Elderly Care Medicine and Institute for Health and Care Research (EMGO+), VU University Medical Centre, Van der Boechorststraat 7, Amsterdam 1081BT, The Netherlands. 'Institute of Psychology, Leiden University, PO Box 9555, Leiden 2300 RB, The Netherlands. ${ }^{7}$ Department of Psychiatry, Leiden University Medical Centre, PO Box 9600, Leiden 2300 RC, The Netherlands.
}

Received: 25 November 2015 Accepted: 23 May 2016

Published online: 02 June 2016

\section{References}

* Studies included in review and meta-analysis

1. Kessler RC, Ruscio AM, Shear K, Wittchen HU. Epidemiology of anxiety disorders. Curr Top Behav Neurosci. 2010;2:21-35.

2. Lepine JP. The epidemiology of anxiety disorders: prevalence and societal costs. J Clin Psychiatry. 2002;63 Suppl 14:4-8.

3. Wang PS, Lane M, Olfson M, Pincus HA, Wells KB, Kessler RC. Twelve-month use of mental health services in the United States: results from the National Comorbidity Survey Replication. Arch Gen Psychiatry. 2005;62:629-40.

4. Young AS, Klap R, Sherbourne CD, Wells KB. The quality of care for depressive and anxiety disorders in the United States. Arch Gen Psychiatry. 2001;58:55-61.

5. Kessler RC, Chiu WT, Demler O, Merikangas KR, Walters EE. Prevalence, severity, and comorbidity of 12-month DSM-IV disorders in the National Comorbidity Survey Replication. Arch Gen Psychiatry. 2005;62:617-27.

6. Fernandez A, Haro JM, Martinez-Alonso M, Demyttenaere K, Brugha TS, Autonell J, et al. Treatment adequacy for anxiety and depressive disorders in six European countries. Br J Psychiatry. 2007;190:172-3.

7. Stein MB, Roy-Byrne PP, Craske MG, Campbell-Sills L, Lang AJ, Golinelli D, et al. Quality of and patient satisfaction with primary health care for anxiety disorders. J Clin Psychiatry. 2011:72:970-6.

8. Smolders M, Laurant M, Verhaak P, Prins M, van Marwijk H, Penninx B, et al. Which physician and practice characteristics are associated with adherence to evidence-based guidelines for depressive and anxiety disorders? Med Care. 2010:48:240-8.

9. Nutting PA, Rost K, Dickinson M, Werner JJ, Dickinson P, Smith JL, et al. Barriers to initiating depression treatment in primary care practice. J Gen Intern Med. 2002;17:103-11.

10. Roy-Byrne PP, Wagner AW, Schraufnagel TJ. Understanding and treating panic disorder in the primary care setting. J Clin Psychiatry. 2005;66 Suppl 4:16-22.

11. Roy-Byrne PP, Wagner A. Primary care perspectives on generalized anxiety disorder. J Clin Psychiatry. 2004;65 Suppl 13:20-6.

12. Gilbody S, Whitty P, Grimshaw J, Thomas R. Educational and organizational interventions to improve the management of depression in primary care: a systematic review. Jama. 2003;289:3145-51.

13. Heideman J, van Rijswijk E, van Lin N, de Loos S, Laurant M, Wensing M, et al. Interventions to improve management of anxiety disorders in general practice: a systematic review. Br J Gen Pract. 2005;55:867-74.

14. Katon W, Von KM, Lin E, Simon G. Rethinking practitioner roles in chronic illness: the specialist, primary care physician, and the practice nurse. Gen Hosp Psychiatry. 2001;23:138-44.

15. Craven MA, Bland R. Better practices in collaborative mental health care: an analysis of the evidence base. Can J Psychiatry. 2006;51:7S-2.

16. Gilbody S, Bower P, Fletcher J, Richards D, Sutton AJ. Collaborative care for depression: a cumulative meta-analysis and review of longer-term outcomes. Arch Intern Med. 2006;166:2314-21.

17. van Steenbergen-Weijenburg KM, van der Feltz-Cornelis CM, Horn EK, van Marwijk HW, Beekman AT, Rutten FF, et al. Cost-effectiveness of collaborative care for the treatment of major depressive disorder in primary care. A systematic review. BMC Health Serv Res. 2010;10:19. Electronic Publication 2010.

18. Von Korff M, Gruman J, Schaefer J, Curry SJ, Wagner EH. Collaborative management of chronic illness. Ann Intern Med. 1997;127:1097-102.

19. Archer J, Bower P, Gilbody S, Lovell K, Richards D, Gask L, et al. Collaborative care for depression and anxiety problems. Cochrane Database Syst Rev. 2012;10:CD006525

20. Woltmann E, Grogan-Kaylor A, Perron B, Georges H, Kilbourne AM, Bauer MS. Comparative effectiveness of collaborative chronic care models for mental health conditions across primary, specialty, and behavioral health care settings: systematic review and meta-analysis. Am J Psychiatry. 2012; 169:790-804.

21. *Oosterbaan DB, Verbraak MJ, Terluin B, Hoogendoorn AW, Peyrot WJ, Muntingh $A$, et al. Collaborative stepped care v. care as usual for common mental disorders: 8-month, cluster randomised controlled trial. $\mathrm{Br} J$ Psychiatry. 2013;203:132-9

22. *Muntingh A, van Mh F-CC, Spinhoven P, Assendelft W, De WM, et al. Effectiveness of collaborative stepped care for anxiety disorders in primary care: a pragmatic cluster randomised controlled trial. Psychother Psychosom. 2014;83:37-44. 
23. Bower P, Gilbody S, Richards D, Fletcher J, Sutton A. Collaborative care for depression in primary care. Making sense of a complex intervention: systematic review and meta-regression. Br J Psychiatry. 2006;189:484-93.

24. Thota AB, Sipe TA, Byard GJ, Zometa CS, Hahn RA, McKnight-Eily LR, et al. Collaborative care to improve the management of depressive disorders: a community guide systematic review and meta-analysis. Am J Prev Med. 2012;42:525-38.

25. Coventry PA, Hudson JL, Kontopantelis E, Archer J, Richards DA, Gilbody S, et al. Characteristics of effective collaborative care for treatment of depression: a systematic review and meta-regression of 74 randomised controlled trials. PLOS ONE. 2014;9:e108114.

26. Liberati A, Altman DG, Tetzlaff J, Mulrow C, GÃ Tzsche PC, loannidis JP, et al. The PRISMA statement for reporting systematic reviews and meta-analyses of studies that evaluate health care interventions: explanation and elaboration. Plos Medicine. 2009;6(7):e1000100. Electronic Publication 2009.

27. Higgins JPT, Deeks JJ, editors. Chapter 7: Selecting studies and collecting data. In: Higgins JPT, Green S, editors. Cochrane Handbook for Systematic Reviews of Interventions Version 5.1.0 (updated March 2011). The Cochrane Collaboration; 2011. Available from www.cochrane-handbook.org.

28. Higgins JPT, Altman DG, Sterne JAC, editors. Chapter 8: Assessing risk of bias in included studies. In: Higgins JPT, Green S, editors. Cochrane Handbook for Systematic Reviews of Interventions Version 5.1.0 (updated March 2011). The Cochrane Collaboration; 2011. Available from www.cochrane-handbook.org.

29. Borenstein M, Hedges LV, Higgins JPT, Rothstein HR: Comprehensive MetaAnalysis, Version 2. Biostat 2005, Englewood NJ, USA.

30. Borenstein M, Hedges LV, Higgins JPT, Rothstein HR. Fixed-effects versus random-effects model (p. 77-85). In: Borenstein M, Hedges LV, Higgins JPT, Rothstein HR. Introduction to Meta-analysis. 1st ed. Chichester (UK): John Wiley \& Sons; 2009.

31. Higgins JP, Thompson SG, Deeks JJ, Altman DG. Measuring inconsistency in meta-analyses. BMJ. 2003;327:557-60.

32. Duval S, Tweedie R. Trim and fill: A simple funnel-plot-based method of testing and adjusting for publication bias in meta-analysis. Biometrics. 2000;56:455-63.

33. *Roy-Byrne PP, Katon W, Cowley DS, Russo J. A randomized effectiveness trial of collaborative care for patients with panic disorder in primary care. Arch Gen Psychiatry. 2001;58:869-76.

34. *Roy-Byrne PP, Craske MG, Stein MB, Sullivan G, Bystritsky A, Katon W, et al. A randomized effectiveness trial of cognitive-behavioral therapy and medication for primary care panic disorder. Arch Gen Psychiatry. 2005;62:290-8.

35. *Rollman BL, Belnap BH, Mazumdar S, Houck PR, Zhu F, Gardner W, et al. A randomized trial to improve the quality of treatment for panic and generalized anxiety disorders in primary care. Arch Gen Psychiatry. 2005;62:1332-41.

36. *Konig HH, Born A, Heider D, Matschinger H, Heinrich S, Riedel-Heller SG, et al. Cost-effectiveness of a primary care model for anxiety disorders. $\mathrm{Br} J$ Psychiatry. 2009;195:308-17.

37. *Roy-Byrne P, Craske MG, Sullivan G, Rose RD, Edlund MJ, Lang AJ, et al. Delivery of evidence-based treatment for multiple anxiety disorders in primary care: a randomized controlled trial. J Am Med Assoc. 2010;303:1921-8.

38. Van der Feltz-Cornelis CM, Ader HJ. Randomization in psychiatric intervention research in the general practice setting. Int J Methods Psychiatr Res. 2000;9:134-42.

39. Craske MG, Roy-Byrne P, Stein MB, Donald-Sherbourne C, Bystritsky A, Katon $W$, et al. Treating panic disorder in primary care: a collaborative care intervention. Gen Hosp Psychiatry. 2002;24(4):148-55.

40. Rollman BL, Herbeck BB, Reynolds CF, Schulberg HC, Shear MK. A contemporary protocol to assist primary care physicians in the treatment of panic and generalized anxiety disorders. Gen Hosp Psychiatry. 2003;25:74-82.

41. Sullivan G, Craske MG, Sherbourne C, Edlund MJ, Rose RD, Golinelli D, et al. Design of the Coordinated Anxiety Learning and Management (CALM) study: innovations in collaborative care for anxiety disorders. Gen Hosp Psychiatry. 2007;29(5):379-87.

42. Muntingh ADT, van der Feltz-Cornelis CM, Van Marwijk HWJ, Spinhoven P, Assendelft WJJ, de Waal MW, et al. Collaborative stepped care for anxiety disorders in primary care: aims and design of a randomized controlled trial. BMC Health Serv Res. 2009;9:159.

43. Sterne JAC, Egger M, Moher B: Addressing reporting bias. In Cochrane handbook for systematic reviews of interventions. Version 5.1.0. Edited by Higgins JPT, Green S. The Cochrane Collaboration; 2011.
44. Craske MG, Stein MB, Sullivan G, Sherbourne C, Bystritsky A, Rose RD, et al. Disorderspecific impact of coordinated anxiety learning and management treatment for anxiety disorders in primary care. Arch Gen Psychiatry. 2011;68:378-88.

45. Seekles W, Cuijpers P, Kok R, Beekman A, van MH, van SA: Psychological treatment of anxiety in primary care: a meta-analysis. Psychol Med. 2012, 1-11.

46. Driessen E, Hollon SD, Bockting CLH, Cuijpers P, Turner EH. Does publication bias inflate the apparent efficacy of psychological treatment for major depressive disorder?: A systematic review and meta-analysis of US National Institutes of Health-Funded Trials. PLOS ONE. 2015;10(9):e0137864. doi: 10.1371/journal.pone.0137864.

47. Rollman B, Mazumdar S, Belnap BH, Houck P, Lenze E, Schulberg H. Main outcomes from the RELAX Trial of telephone-delivered collaborative care for panic and generalized anxiety disorder. J Gen Intern Med. 2010;25:S326.

48. Cuijpers P, Van SA, Van SA, Andersson G. Psychological treatment of depression in primary care: a meta-analysis. Br J Gen Pract. 2009;59:e51-60.

49. van Balkom AJ, van Boeijen CA, Boeke AJ, van Oppen P, Kempe PT, van Dyck R. Comorbid depression, but not comorbid anxiety disorders, predicts poor outcome in anxiety disorders. Depress Anxiety. 2008;25:408-15.

50. Moher D, Liberati A, Tetzlaff J, Altman DG. Preferred reporting items for systematic reviews and meta-analyses: the PRISMA statement. J Clin Epidemiol. 2009;62(10):1006-12. Electronic Publication 2009

\section{Submit your next manuscript to BioMed Central and we will help you at every step:}

- We accept pre-submission inquiries

- Our selector tool helps you to find the most relevant journal

- We provide round the clock customer support

- Convenient online submission

- Thorough peer review

- Inclusion in PubMed and all major indexing services

- Maximum visibility for your research

Submit your manuscript at www.biomedcentral.com/submit

C Biomed Central 